\title{
Probing double parton scattering via associated open charm and bottom production in ultraperipheral $p A$ collisions
}

\author{
Edgar Huayra $^{1, \mathrm{a}}$, Emmanuel G. de Oliveira ${ }^{1, \mathrm{~b}}$, Roman Pasechnik ${ }^{1,2,3, \mathrm{c}}$ \\ ${ }^{1}$ Departamento de Física, CFM, Universidade Federal de Santa Catarina, C.P. 476, Florianópolis, SC CEP 88040-900, Brazil \\ 2 Department of Astronomy and Theoretical Physics, Lund University, 22362 Lund, Sweden \\ ${ }^{3}$ Nuclear Physics Institute ASCR, 25068 Řež, Czech Republic
}

Received: 10 May 2019 / Accepted: 10 October 2019 / Published online: 26 October 2019

(C) The Author(s) 2019

\begin{abstract}
In this article, we propose a novel channel for phenomenological studies of the double-parton scattering (DPS) based upon associated production of charm $c \bar{c}$ and bottom $b \bar{b}$ quark pairs in well-separated rapidity intervals in ultraperipheral high-energy proton-nucleus collisions. This process provides direct access to the double-gluon distribution in the proton at small- $x$ and enables one to test the factorized DPS pocket formula. We have made the corresponding theoretical predictions for the DPS contribution to this process at typical LHC energies and beyond and we compute the energy-independent (but photon momentum fraction dependent) effective cross section.
\end{abstract}

\section{Introduction}

With an increase of collision energy, the probability for more than one parton-parton scattering to occur in the same proton-proton or proton-nucleus collision grows faster compared with that of the single-parton scattering (SPS) leading to the well-known phenomenon of multi-parton interactions (MPIs) known since a long time ago [1-3]. Due to measurements at the Large Hadron Collider (LHC), the physics of MPIs has attracted a lot of attention from both theoretical and experimental communities (for recent work on this topic, see e.g. Refs. [4-12] and the references therein). A first non-trivial example, the double-parton scattering (DPS), becomes particularly significant in production of specific multi-particle final states such as meson pairs [10], four identified jets [11] or leptons [12] etc. These processes are traditionally considered as an important source of information about a new class of non-perturbative QCD objects,

\footnotetext{
a e-mail: yuberth022@gmail.com

b e-mail: emmanuel.de.oliveira@ufsc.br

c e-mail: Roman.Pasechnik@thep.lu.se
}

the double-parton distribution functions (dPDFs) being now actively explored in the literature. They describe the number density and correlations of two colored partons in the proton, with given longitudinal momentum fractions $x_{1}, x_{2}$ and placed at a given transverse relative separation $\mathbf{b}$ of the two hard collisions [13] (for a detailed review on theoretical grounds, see e.g. Ref. [14] and the references therein).

While complete theoretical predictions for dPDFs involving the unknown non-perturbative QCD parton correlation functions are not available, a few model calculations exist attempting to pick the most significant features of dPDFs $[8,9,15-17]$. In order to perform any comprehensive verification of such models, much more phenomenological information is needed as no direct measurement or extraction of dPDFs from the experimental data has yet been possible. Experimentally, a distinctive signature of DPS associated with the so-called effective cross section, $\sigma_{\text {eff }}$, has already been identified and measured in different channels at central rapidities (see e.g. Refs. [11,12,18-24]), while many MonteCarlo generators naturally incorporate MPIs as part of their framework [3].

The effective cross section is experimentally defined as ratio of double to product of two single inclusive production rates for final-state $A_{1}$ and $A_{2}$ systems in two independent hard scatterings and represents the effective transverse overlap area containing the interacting hard partons. With this definition, the DPS cross section is estimated as $[5,6,25]$ (for a detailed review on this topic, see e.g. Refs. [26,27]),

$\sigma_{\mathrm{DPS}}^{A_{1} A_{2}}=\frac{\kappa}{2} \frac{\sigma_{\mathrm{SPS}}^{A_{1}} \sigma_{\mathrm{SPS}}^{A_{2}}}{\sigma_{\mathrm{eff}}}$,

where $\sigma_{\mathrm{SPS}}^{A_{1,2}}$ represents the corresponding SPS cross section for production of $A_{1,2}$ systems, and $\kappa$ is the symmetry factor depending on whether the final states are the same $\left(A_{1}=A_{2}\right.$, $\kappa=1)$ or different $\left(A_{1} \neq A_{2}, \kappa=2\right)$. In general, $\sigma_{\text {eff }}$ depends on scales, momentum fractions, and parton flavors 
Fig. 1 A schematic illustration of the $A+p \rightarrow A+(c \bar{c} b \bar{b})+X$ cross section in $p A$ UPCs

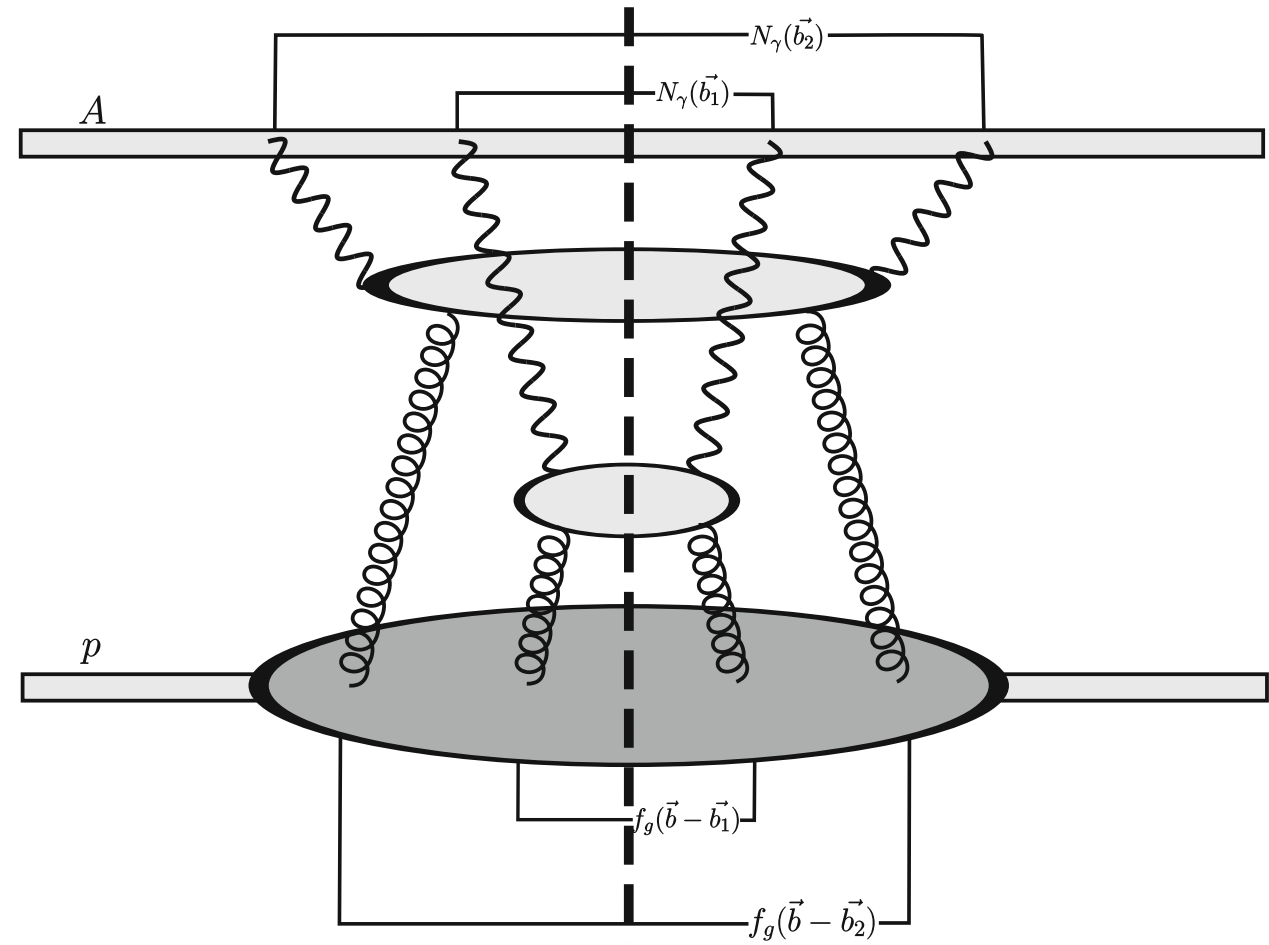

involved. In many theoretical studies it is, however, assumed that it is a constant geometrical factor; under this approximation (1.1) is known as the "pocket formula".

Among the hadron final states, double open heavy flavor production is considered to be an important and promising tool for probing the DPS mechanism [28]. In particular, the LHCb Collaboration has recently reported an enhancement in the data on double charm production cross section in $p p$ collisions $[20,29]$ that could not be described without a significant DPS contribution as was found in Ref. [30]. More possibilities have been recently discussed also in the case of $c \bar{c} b \bar{b}$ and $b \bar{b} b \bar{b}$ final states, as well as in associated production of open heavy flavor and jets, in Refs. [10,31-33].

Within yet large experimental uncertainties, the c.m. collision energy dependence of the effective cross section is consistent with a constant $\sigma_{\text {eff }} \sim 15-20 \mathrm{mb}$ for the channels probed by most of the existing measurements $[11,12,18$ 24]. However, in associated production of heavy quarkonia such as double- $J / \psi$ and $J / \psi \Upsilon$, one discovers systematically lower values of $\sigma_{\text {eff }}$ than in all the other channels studied so far [34-37]. Such a discrepancy may hint towards a non-universality of $\sigma_{\text {eff }}$ due to e.g. spatial fluctuations of the parton densities [38]. Typically, measurements of the DPS contributions for different production processes need a dedicated experimental analysis and tools, and the precision is usually very limited and suffers due to large backgrounds coming from the standard SPS processes.

The use of ultraperipheral $p A$ collisions (UPCs) for probing the DPS mechanism and further constraining the effective cross section has not yet been properly studied in the literature. In contrast, the SPS UPC case has been studied in, e.g., Refs. $[39,40]$. In UPCs, the high-energy colliding systems pass each other at large transverse separations and thus do not undergo hadronic interactions. In this case, they interact electromagnetically via an exchange of quasi-real photons. The corresponding Weiszäcker-Williams (WW) photon flux $[41,42]$ is scaled with the square of electric charge of the emitter and is thus strongly enhanced for a heavy nucleus making the $p A$ and $A A$ UPCs more advantageous compared with that in $p p$ collisions. It is worth noticing that the photon spectrum of a heavy nucleus is rather broad, where the peak-energy in the target rest frame scales linearly with the nuclear Lorentz factor which represents yet another advantage of UPCs. Finally, an additional reduction of the backgrounds is provided by tagging on the final-state nucleus identifying the momentum transfer taken by the exchanged photon, together with reconstructing the four-momenta of the produced final-state particles.

In this work, we explore possibilities for a new measurement of the gluon dPDF in the proton at small- $x$ by means of $A+p \rightarrow A+(c \bar{c} b \bar{b})+X$ reaction in high-energy $p A$ UPCs schematically illustrated in Fig. 1. This process offers interesting possibilities and a cleaner environment for probing the DPS contribution compared with that in $p p$ collisions.

In order to study the corresponding reaction in UPCs, we have to compute an effective cross section for the interaction of two partons (e.g. gluons) on one side with two photons on the other side, in contrast to the four parton case in reg- 
ular $p p$ or $p A$ collisions. At small $x$ and for hadronic final states, these partons are overwhelmingly likely to be gluons, so in the subsequent discussion we will simply refer to them as gluons. By exchanging the two-gluon initial state by two photons on the nucleus side, the effective cross section is expected to increase significantly. This is due to the fact that two photons from a single nucleus overlap much less than the two gluons, since the latter are well localized inside the nucleons, while photons are more spread out, specially in the case of UPCs, when they are required to be outside the nucleus. As far as we know, this effective cross section has not yet been calculated or measured earlier, but it is clearly important in order to better understand the impact-parameter dependence of parton distributions in general.

Regarding the order in coupling constants, the DPS process is of the order of $\left(\alpha \alpha_{s}\right)^{2}$, while the SPS process- $\alpha \alpha_{s}^{3}$, i.e. the SPS cross section is by default a factor of $\alpha_{s} / \alpha$ larger. However, the DPS reaction is expected to dominate the $c \bar{c} b \bar{b}$ production cross section over the SPS one at high energies, particularly, for a large separation between rapidities of $c \bar{c}$ and $b \bar{b}$ pairs. Indeed, in the case of a large invariant mass of the $c \bar{c} b \bar{b}$ system, the parton distributions are computed at larger $x$ for the SPS case than that in DPS, since more energy in the initial state is needed, especially if there is a considerably large rapidity difference between the $c \bar{c}$ and $b \bar{b}$ pairs. As the PDFs decrease very fast with $x$ in the case of gluons (and photons likewise), the SPS process is expected to be suppressed.

Thus, in order to extract the DPS contribution to this process, one should consider light $c \bar{c}$ and $b \bar{b}$ pairs produced at relatively large rapidity separation $\delta Y=Y_{c \bar{c}}-Y_{b \bar{b}} \gg 1$. This is required in order to maximize the invariant mass of the SPS $\gamma+g \rightarrow c \bar{c} b \bar{b}$ background process, and hence to sufficiently suppress the background compared with the DPS contribution whose dependence on $\delta Y$ is expected to be flatter. In the case of $c \bar{c} c \bar{c}$ and $b \bar{b} b \bar{b}$ production, however, such a separation would be much more difficult (if not impossible), since combining a quark $Q$ and antiquark $\bar{Q}$ of the same flavor does not guarantee that they come from the same SPS process $\gamma+g \rightarrow Q \bar{Q}$. The relative $\delta P_{\perp}=P_{\perp}^{c \bar{c}}-P_{\perp}^{b \bar{b}}$ variable is of less importance for the SPS background suppression since both the SPS and the DPS components are peaked around a small $\delta P_{\perp} \approx 0$, while at large $\delta P_{\perp}$ the DPS term is nonzero only at the NLO level.

The relative SPS background suppression at large $\delta Y$ is only a qualitative expectation based upon simple kinematical arguments mentioned above. In this work, however, we focus only on the DPS contribution to the $c \bar{c}$ and $b \bar{b}$ pair production. The detailed analysis of the SPS $\gamma+g \rightarrow c \bar{c} b \bar{b}$ amplitude and the corresponding differential cross section falls beyond the scope of the current work and will be performed elsewhere.
The paper is organized as follows. In Sect. 2 we derive the formula for the UPC double heavy quark photoproduction, which is written with the help of an effective cross section that depends on the photon longitudinal momentum fraction. We also review the key components of such a calculation. In Sect. 3 we present our numerical results at LHC and larger energies. We conclude our paper in Sect. 4.

\section{Double quark-pair production in UPC: DPS mechanism}

In the high-energy limit, the cross section for $c \bar{c} b \bar{b}$ production via DPS can be represented as a convolution of the impact-parameter dependent differential probabilities to produce separate $c \bar{c}$ and $b \bar{b}$ pairs in $p A$ collisions,

$$
\begin{aligned}
& \frac{d^{4} \sigma_{p A \rightarrow X A+c \bar{c}+b \bar{b}}}{d y_{c} d y_{\bar{c}} d y_{b} d y_{\bar{b}}} \\
& =\int d^{2} \vec{b} \Theta\left(b-R_{A}-R_{p}\right) \frac{d^{2} P_{p A \rightarrow X A+c \bar{c}}(b)}{d y_{c} d y_{\bar{c}}} \\
& \quad \times \frac{d^{2} P_{p A \rightarrow X A+b \bar{b}}(b)}{d y_{b} d y_{\bar{b}}} .
\end{aligned}
$$

Here, $R_{A}$ and $R_{p}$ are the nuclei and the proton radii, respectively, $\vec{b}$ is the relative impact parameter $(b \equiv|\vec{b}|)$. The $\Theta$ function represents an approximate absorption factor, which ensures that one considers only peripheral collisions when no nucleus break-up occurs [43]. Let us consider the ingredients of the DPS cross section (2.1) in detail.

\subsection{SPS subprocess cross section}

The differential probabilities $P(b)$ in Eq. (2.1) can be deduced from the corresponding SPS cross sections. For instance, for SPS production of the $c \bar{c}$ pair we have

$$
\begin{aligned}
& \frac{d^{3} \sigma_{p A \rightarrow X A+c \bar{c}}}{d y_{c} d y_{\bar{c}} d p_{\perp}^{2}} \\
& \quad=\int d^{2} \vec{b} \Theta\left(b-R_{A}-R_{p}\right) \frac{d^{3} P_{p A \rightarrow X A+c \bar{c}}(b)}{d y_{c} d y_{\bar{c}} d p_{\perp}^{2}}
\end{aligned}
$$

where

$$
\begin{aligned}
& \frac{d^{3} P_{p A \rightarrow X A+c \bar{c}}(b)}{d y_{c} d y_{\bar{c}} d p_{\perp}^{2}}=\int d^{2} \vec{b}_{\gamma} d^{2} \vec{b}_{g} \delta^{(2)}\left(\vec{b}+\vec{b}_{g}-\vec{b}_{\gamma}\right) \\
& \times \int d \xi d x N_{\gamma}\left(\xi, \vec{b}_{\gamma}\right) G_{g}\left(x, \vec{b}_{g}\right) \mathcal{J} \frac{d^{3} \hat{\sigma}_{\gamma g \rightarrow c \bar{c}}}{d y_{c} d y_{\bar{c}} d \hat{t}}
\end{aligned}
$$


in terms of the differential parton-level $\gamma+g \rightarrow c \bar{c}$ cross section,

$$
\begin{aligned}
\frac{d^{3} \hat{\sigma}_{\gamma g \rightarrow c \bar{c}}}{d y_{c} d y_{\bar{c}} d \hat{t}}= & \frac{d \hat{\sigma}_{\gamma g \rightarrow Q \bar{Q}}}{d \hat{t}} \delta\left(y_{c}-\frac{1}{2} \ln \left(\frac{\xi}{x} \frac{\hat{u}}{\hat{t}}\right)\right) \delta \\
& \times\left(y_{\bar{c}}-\frac{1}{2} \ln \left(\frac{\xi}{x} \frac{\hat{t}}{\hat{u}}\right)\right),
\end{aligned}
$$

written with the help of the modified Mandelstam variable

$$
\hat{t}=\left(p_{c}-p_{\gamma}\right)^{2}-m_{Q}^{2}=-\sqrt{\hat{s}}\left(\frac{\sqrt{\hat{s}}}{2}-\sqrt{\frac{\hat{s}}{4}-m_{Q}^{2}-p_{\perp}^{2}}\right),
$$

and the Jacobian is

$\mathcal{J} \equiv\left|\frac{d \hat{t}}{d p_{\perp}^{2}}\right|=\frac{\sqrt{\hat{s}}}{2 \sqrt{\frac{\hat{s}}{4}-m_{Q}^{2}-p_{\perp}^{2}}}$

as well as the photon $N_{\gamma}\left(\xi, \vec{b}_{\gamma}\right)$ and gluon $G_{g}\left(x, \vec{b}_{g}\right)$ distributions for the longitudinal momentum fractions $\xi, x$ and impact parameters $\vec{b}_{\gamma}$ and $\vec{b}_{g}$, respectively. The elementary cross section for the direct (fusion) subprocess reads in terms of the Mandelstam variables of the subprocess

$$
\begin{aligned}
& \frac{d^{2} \hat{\sigma}_{\gamma g \rightarrow Q \bar{Q}}}{d \hat{t} d \hat{u}}=\frac{\pi \alpha_{s} \alpha e_{Q}^{2}}{\hat{s}^{2}} \\
& \quad \times\left[\frac{\hat{t}}{\hat{u}}+\frac{\hat{u}}{\hat{t}}+\frac{4 m_{Q}^{2} \hat{s}}{\hat{t} \hat{u}}\left(1-\frac{m_{Q}^{2} \hat{s}}{\hat{t} \hat{u}}\right)\right] \delta(\hat{s}+\hat{t}+\hat{u}) .
\end{aligned}
$$

Provided that the quark mass regulates the infrared behavior of the integrals, there is no need to introduce additional low$p_{t}$ cuts in order to unitarize the probabilities $P_{p A \rightarrow Q \bar{Q}}$ as for the heavy quarks they are below unity.

The standard WW photon flux is determined as

$$
\frac{d^{3} N_{\gamma}(\omega, \vec{b})}{d \omega d^{2} \vec{b}}=\frac{Z^{2} \alpha k^{2}}{\pi^{2} \omega b^{2}}\left[K_{1}^{2}(k)+\frac{1}{\gamma^{2}} K_{0}^{2}(k)\right], \quad k=\frac{b \omega}{\gamma},
$$

with the nucleus charge $Z$, the modified Bessel functions of the second kind $K_{0}$ and $K_{1}$, the fine structure constant $\alpha$, the photon energy $\omega$, the Lorentz factor $\gamma$ defined as, $\gamma=\sqrt{s} / 2 m_{p}$, where $s$ is the center-of-mass (c.m.) energy per nucleon, and the proton mass $m_{p}=0.938 \mathrm{GeV}$. For instance, at LHC $p A 2016$ run (with $\sqrt{s}=8.16 \mathrm{TeV}$ ) we have $\gamma_{\mathrm{Pb}} \approx 4350$, while for RHIC, $\gamma_{\mathrm{Au}} \approx 107$. For FCC collider (with $\sqrt{s}=50 \mathrm{TeV}$ ), we have $\gamma \approx 26652$. Since we would like to work with the photon momentum fraction instead of photon energy, we have

$\frac{d^{3} N_{\gamma}(\xi, \vec{b})}{d \xi d^{2} \vec{b}}=\frac{\sqrt{s}}{2} \frac{d^{3} N_{\gamma}(\omega, \vec{b})}{d \omega d^{2} \vec{b}} \quad$ with $\quad \xi=\frac{2 \omega}{\sqrt{s}}$.
Another important ingredient is the impact-parameter dependent gluon distribution $G_{g}(x, \vec{b})$, which is often used in a factorized form,

$G_{g}(x, \vec{b})=g(x) f_{g}(\vec{b})$,

where $g(x)$ is the usual integrated gluon PDF, with an implicit factorization scale dependence, and $f_{g}(b)$ is the normalized spatial gluon distribution in the transverse plane

$f_{g}(\vec{b})=\frac{\Lambda^{2}}{2 \pi} \frac{\Lambda b}{2} K_{1}(\Lambda b), \quad \int d^{2} \vec{b} f_{g}(\vec{b})=1$,

as in Ref. [44]. Here, $\Lambda \approx 1.5 \mathrm{GeV}$ and the CT14nlo [45] collinear parton distributions are used with $\mu_{F}=\hat{s}$.

Consequently, the cross section for $c \bar{c}$ production in the SPS UPCs is related to the parton-level $\gamma+g \rightarrow c \bar{c}$ cross section as follows:

$$
\begin{aligned}
\frac{d^{2} \sigma_{p A \rightarrow X A+c \bar{c}}}{d y_{c} d y_{\bar{c}}}= & \int d^{2} \vec{b} d^{2} \vec{b}_{\gamma} d^{2} \vec{b}_{g} d \xi d x \\
& \times \Theta\left(b-R_{A}-R_{p}\right) \delta^{(2)}\left(\vec{b}+\vec{b}_{g}-\vec{b}_{\gamma}\right) \\
& \times N_{\gamma}\left(\xi, \vec{b}_{\gamma}\right) G_{g}\left(x, \vec{b}_{g}\right) \frac{d^{2} \hat{\sigma}_{\gamma g \rightarrow c \bar{c}}}{d y_{c} d y_{\bar{c}}} .
\end{aligned}
$$

This expression can be rewritten in the following equivalent form:

$$
\begin{aligned}
& \frac{d^{2} \sigma_{p A \rightarrow X A+c \bar{c}}}{d y_{c} d y_{\bar{c}}}=\int d \xi \int d x \bar{N}_{\gamma}(\xi) g(x) \frac{d^{2} \hat{\sigma}_{\gamma g \rightarrow c \bar{c}}}{d y_{c} d y_{\bar{c}}} \\
& \times \int d^{2} \vec{b} \Theta\left(b-R_{A}-R_{p}\right) T_{g \gamma}(\xi, \vec{b})
\end{aligned}
$$

with the overlap function, which encapsulates all the impactparameter dependence in the matrix element squared, defined as follows:

$$
\begin{aligned}
& T_{g \gamma}(\xi, \vec{b})=\frac{1}{\bar{N}_{\gamma}(\xi)} \int d^{2} \vec{b}_{\gamma} \Theta\left(b_{\gamma}-R_{A}\right) \\
& \quad \times N_{\gamma}\left(\xi, \vec{b}_{\gamma}\right) f_{g}\left(\vec{b}-\vec{b}_{\gamma}\right)
\end{aligned}
$$

where

$\bar{N}_{\gamma}(\xi)=\int d^{2} b \Theta\left(b-R_{A}\right) N_{\gamma}(\xi, \vec{b})$

is the number distribution of photons that can interact in the considering process (outside the nucleus) shown in Fig. 2. Here, it becomes apparent that the photon distribution is strongly peaked at low $\xi<10^{-2}$. The distribution calculated with the WW flux is independent of energy if the Lorentz factor is very large, $\gamma \rightarrow \infty$. 


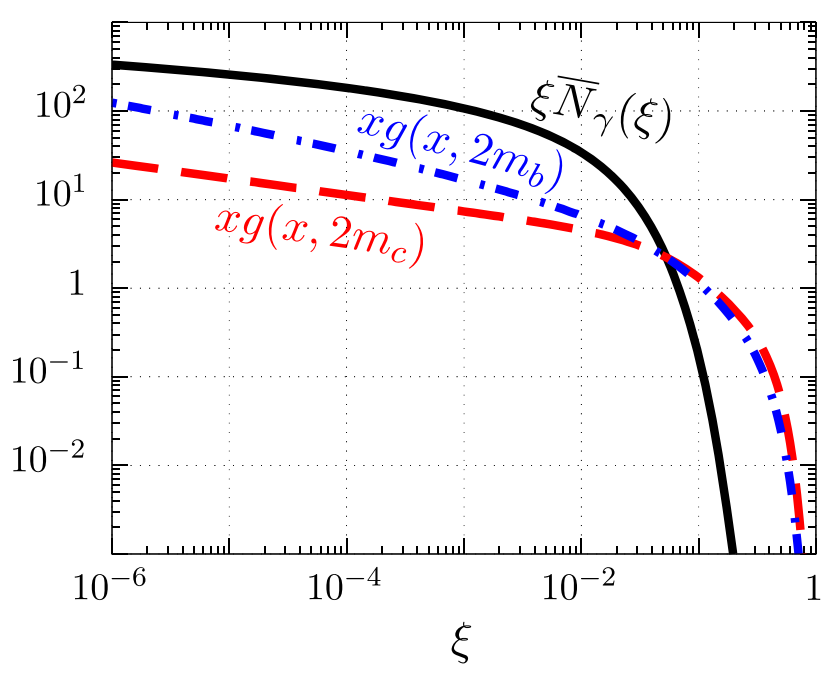

Fig. 2 The number distribution of photons $\bar{N}_{\gamma}(\xi)$ (times $\xi$ ) outside the nucleus that can interact with gluons in the proton in the considering photoproduction process. For comparison, the gluon distributions at two factorization scales $2 m_{c}$ and $2 m_{b}$

It is worth noticing that the last integral in Eq. (2.13) can be transformed as follows:

$$
\begin{aligned}
\int & d^{2} \vec{b} \Theta\left(b-R_{A}-R_{p}\right) T_{g \gamma}(\xi, \vec{b}) \\
= & \int d^{2} \vec{b} \frac{\Theta\left(b-R_{A}-R_{p}\right)}{\bar{N}_{\gamma}(\xi)} \int d^{2} \vec{b}_{\gamma} \Theta\left(b_{\gamma}-R_{A}\right) \\
& \times N_{\gamma}\left(\xi, \vec{b}_{\gamma}\right) f_{g}\left(\vec{b}_{\gamma}-\vec{b}\right) \\
= & 1-\int d^{2} \vec{b} \int d^{2} \vec{b}_{\gamma} \\
& \times \frac{\Theta\left(b_{\gamma}-R_{A}\right)\left(1-\Theta\left(b-R_{A}-R_{p}\right)\right)}{\bar{N}_{\gamma}(\xi)} \\
& \times N_{\gamma}\left(\xi, \vec{b}_{\gamma}\right) f_{g}\left(\vec{b}_{\gamma}-\vec{b}\right) .
\end{aligned}
$$

This relation explicitly demonstrates that, if the gluon distribution is very localized, i.e. $R_{p} \rightarrow 0$ together with $b_{\gamma} \rightarrow b$, the second term vanishes, leaving no traces of the impactparameter dependence in the SPS cross section (2.13).

For completeness, besides the direct production process whose formalism is discussed above, we have also included a sub-dominant resolved contribution following Ref. [39]. By including the resolved component with the gluon-initiated hard subprocess $g g \rightarrow Q \bar{Q}$ at leading order (LO), as an example, one is enabled to pick a gluon (with momentum fraction $z$ ) from the incident photon by means of a gluon PDF in the photon, $g^{\gamma}\left(z, \mu^{2}\right)$, while the photon remnant hadronizes into an unobserved hadronic system. The corresponding contribution reads

$$
\begin{aligned}
\frac{d^{2} \sigma_{p A \rightarrow X A+c \bar{c}}^{\text {Resolved, gluon }}}{d y_{c} d y_{\bar{c}}}= & \int d \xi d x \bar{N}_{\gamma}(\xi) g(x) \\
& \times \int d z g^{\gamma}(z) \frac{d^{2} \hat{\sigma}_{g g \rightarrow Q \bar{Q}}}{d y_{c} d y_{\bar{c}}} \\
& \times \int d^{2} \vec{b} \Theta\left(b-R_{A}-R_{p}\right) T_{g \gamma}(\xi, \vec{b}) .
\end{aligned}
$$

In our numerical analysis, we also include the second relevant subprocess $q \bar{q} \rightarrow Q \bar{Q}$ at LO. The PDFs in the photon are taken from Ref. [46]. Such a resolved photon contribution increases the differential cross section only slightly and mostly at negative rapidities (for more details and the corresponding figures, see below).

\subsection{Pocket formula for the DPS cross section in $p A$ UPCs}

Consider now the formalism for the DPS mechanism of direct $c \bar{c} b \bar{b}$ production in $p A$ UPCs (while the resolved photon contributions to the DPS are also included into the numerical analysis).

In what follows, we define the nucleus with positive rapidity, and the proton with negative one, such that the longitudinal photon and gluon momentum fractions are given by

$$
\begin{gathered}
\xi_{i}=\frac{m_{i, \perp}}{\sqrt{s}}\left(e^{y_{Q_{i}}}+e^{y} \bar{Q}_{i}\right), \quad x_{i}=\frac{m_{i, \perp}}{\sqrt{s}}\left(e^{-y_{Q_{i}}}+e^{-y_{Q_{i}}}\right), \\
m_{i, \perp}^{2}=m_{Q_{i}}^{2}+p_{i, \perp}^{2},
\end{gathered}
$$

respectively, where the index $i=1,2$ denotes the elementary SPS processes or, equivalently, the heavy quark species the photon and gluon are coupled to, namely, $Q_{1,2} \equiv c, b$ in the process considered, $m_{i, \perp}$ is the transverse mass of the heavy quark, $y_{Q_{i}}\left(y_{\bar{Q}_{i}}\right)$ is the heavy quark (antiquark) rapidity. Then the invariant mass of each $Q_{i} \bar{Q}_{i}$ pair reads

$M_{i, Q \bar{Q}}^{2}=2 x_{i} \xi_{i} s=2 m_{i, \perp}^{2}\left(1+\cosh \left(y_{Q_{i}}-y_{\bar{Q}_{i}}\right)\right)$

such that

$d x_{i} d \xi_{i}=\frac{m_{i, \perp}^{2}}{s}\left|\sinh \left(y_{Q_{i}}-y_{\bar{Q}_{i}}\right)\right| d y_{Q_{i}} d y_{\bar{Q}_{i}}$

Consequently, the DPS cross section (2.1) can be rewritten in terms of parton-level elementary photon-gluon fusion cross sections as 


$$
\begin{aligned}
& \frac{d^{4} \sigma_{p A \rightarrow X A+c \bar{c}+b \bar{b}}}{d y_{c} d y_{\bar{c}} d y_{b} d y_{\bar{b}}}=\int d^{2} b \Theta\left(b-R_{A}-R_{p}\right) \\
& \times \int d^{2} \vec{b}_{\gamma, 1} \Theta\left(b_{\gamma, 1}-R_{A}\right) \\
& \quad \times \int d^{2} \vec{b}_{\gamma, 2} \Theta\left(b_{\gamma, 2}-R_{A}\right) \\
& \quad \times \int d \xi_{1} d \xi_{2} d x_{1} d x_{2} N_{\gamma \gamma}\left(\xi_{1}, \vec{b}_{\gamma, 1} ; \xi_{2}, \vec{b}_{\gamma, 2}\right) \\
& \quad \times G_{g g}\left(x_{1}, \vec{b}_{g, 1} ; x_{2}, \vec{b}_{g, 2}\right) \frac{d^{2} \hat{\sigma}_{\gamma g \rightarrow c \bar{c}}}{d y_{c} d y_{\bar{c}}} \frac{d^{2} \hat{\sigma}_{\gamma g \rightarrow b \bar{b}}}{d y_{b} d y_{\bar{b}}}
\end{aligned}
$$

where $\vec{b}_{g, i}=\vec{b}_{\gamma, i}-\vec{b}$ for $i=1,2$, and $N_{\gamma \gamma}\left(G_{g g}\right)$ is the corresponding di-photon (di-gluon) distribution.

If we neglect any correlations between the individual photon and gluon exchanges, the di-photon and di-gluon distributions are conveniently represented in a factorized form, i.e.

$$
\begin{aligned}
N_{\gamma \gamma}\left(\xi_{1}, \vec{b}_{\gamma, 1} ; \xi_{2}, \vec{b}_{\gamma, 2}\right) & =N_{\gamma}\left(\xi_{1}, \vec{b}_{\gamma, 1}\right) N_{\gamma}\left(\xi_{2}, \vec{b}_{\gamma, 2}\right) \\
G_{g g}\left(x_{1}, \vec{b}_{g, 1} ; x_{2}, \vec{b}_{g, 2}\right) & =G_{g}\left(x_{1}, \vec{b}_{g, 1}\right) G_{g}\left(x_{2}, \vec{b}_{g, 2}\right),
\end{aligned}
$$

in terms of the quasi-real single photon $N_{\gamma}(\xi, \vec{b})$ and gluon $G_{g}(x, \vec{b})$ distributions defined above. Note that the above factorization formulas are approximations valid for $\xi_{1,2}, x_{1,2} \ll$ 1 only [4,9,47-49].

Using Eqs. (2.20), (2.10), (2.11) and (2.14), it is straightforward to transform the resulting DPS cross section to the following simple form:

$$
\begin{aligned}
& \frac{d^{4} \sigma_{p A \rightarrow X A+c \bar{c}+b \bar{b}}}{d y_{c} d y_{\bar{c}} d y_{b} d y_{\bar{b}}} \\
& =\int d \xi_{1} d x_{1} d \xi_{2} d x_{2} \frac{\bar{N}_{\gamma}\left(\xi_{1}\right) g\left(x_{1}\right) \bar{N}_{\gamma}\left(\xi_{2}\right) g\left(x_{2}\right)}{\sigma_{\mathrm{eff}}\left(\xi_{1}, \xi_{2}\right)} \\
& \quad \times \frac{d^{2} \hat{\sigma}_{\gamma g \rightarrow c \bar{c}}}{d y_{c} d y_{\bar{c}}} \frac{d^{2} \hat{\sigma}_{\gamma g \rightarrow b \bar{b}}}{d y_{b} d y_{\bar{b}}}
\end{aligned}
$$

where $\bar{N}_{\gamma}(\xi)$ is defined in Eq. (2.15), and the definition of the effective cross section,

$$
\begin{aligned}
& \sigma_{\mathrm{eff}}\left(\xi_{1}, \xi_{2}\right) \\
& \quad \equiv\left[\int d^{2} b \Theta\left(b-R_{A}-R_{p}\right) T_{g \gamma}\left(\xi_{1}, b\right) T_{g \gamma}\left(\xi_{2}, b\right)\right]^{-1}
\end{aligned}
$$

has been introduced. In this way we arrive at an analog of the pocket formula applicable for the DPS contribution to $c \bar{c} b \bar{b}$ production in $p A$ UPCs. Equation (2.21) is valid also for the DPS contribution to the $c \bar{c} c \bar{c}$ and $b \bar{b} b \bar{b}$ production processes apart from the change in the symmetry factor.

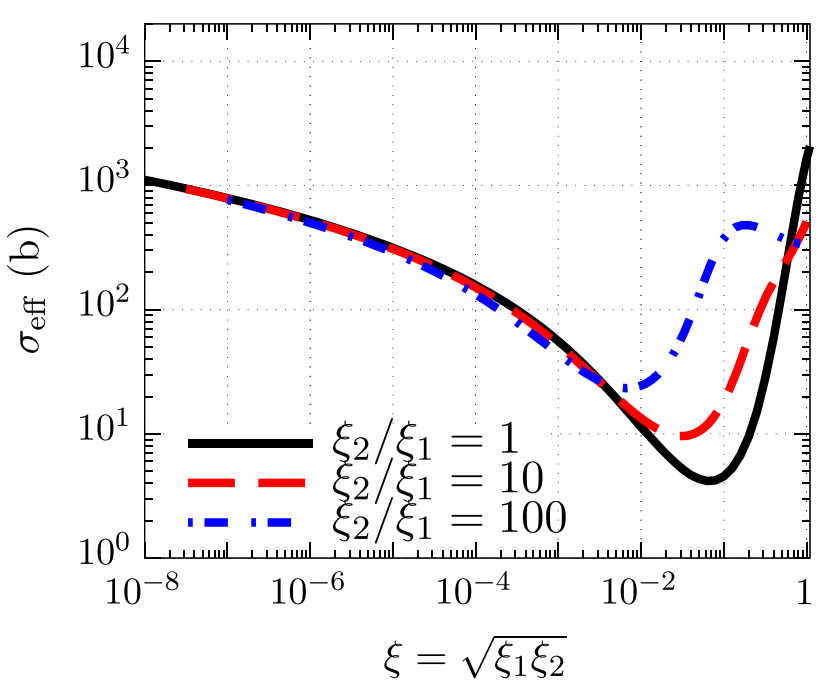

Fig. 3 The DPS effective cross section as a function of $\sqrt{\xi_{1} \xi_{2}}$

In what follows, we wish to investigate the corresponding SPS and DPS differential (in rapidity) cross sections making predictions for future measurements.

\section{Numerical results}

In our numerical analysis of the SPS and DPS cross sections for heavy flavor production in $p A$ UPCs, we consider lead nucleus, with radius $R_{\mathrm{Pb}}=5.5 \mathrm{fm}$ (the proton radius is fixed to $R_{p}=0.87 \mathrm{fm}$ ), while the heavy quark masses are taken to be $m_{c}=1.4 \mathrm{GeV}$ and $m_{b}=4.75 \mathrm{GeV}$.

In Fig. 3 the effective cross section for pA UPCs of Eq. (2.22) is plotted as a function of photon momentum fraction $\xi$. This plot carries essentially the information of where the photons are, but not of the number of photons outside the nucleus, as it is factored out in $\bar{N}_{\gamma}(\xi)$. The main contribution to this result arises when the two photons are inside the proton. With the model used here, the plot does not change with energy or factorization scale.

Take first the case when $\xi_{1}=\xi_{2}$. For small $\xi$, the photons are spread too much and it is more rare that they overlap; then the effective cross section is larger. For large $\xi$, the photons are in a narrow shell just outside the nucleus, and if the width of this shell is smaller than the proton radius, it is clear that $\sigma_{\text {eff }}$ should also grow. That explains the minimum around $\xi \approx 0.07$, where half of the photons outside the nucleus have $b_{\gamma}-R_{\mathrm{Pb}}<1.0 \mathrm{fm}$, i.e., approximately the proton radius.

In the case of $\xi_{2} / \xi_{1}>1, \sigma_{\text {eff }}$ can have two minima, as shown in the plot. That happens because the two photon distributions have their maximum probability of finding the photons inside the proton at different $\xi$.

To better understand our double parton results, we recalculate the SPS cross section in Fig. 4. We show the differential 
cross section in $c$ quark rapidity, for energies of $8.16 \mathrm{TeV}$ and $50 \mathrm{TeV}$. The heavy ion comes from the left, while the proton comes from the right.

We detail the direct and the resolved contributions to the result. The resolved contribution is only relevant at small $z \xi$ and large $x$. Indeed, we see a harder decrease at positive rapidities than at negative rapidities, due to the nucleus photons having a sharper cutoff when $\xi \rightarrow 1$ than the proton gluons. The curves are almost flat at central rapidities; the resolved contribution makes it even flatter due to a small modification to the shape of the resulting differential cross section (mostly) in the negative rapidity domain. While the relative importance of the resolved contribution grows with energy, it remains minor compared with the direct one; see also Table 1 .

For double parton scattering, we present, in Fig. 5, the $c \bar{c} b \bar{b}$ cross section with $y_{\bar{c}}, y_{\bar{b}}$ integrated out but differential in $y_{c}, y_{b}$ (at left and right panels, respectively). The production at central rapidities increases with rapidity; this is in contrast with the SPS case where it was flat. If the DPS was just the product of two SPS cross sections, we would not see such an increase. In effect, this is a result of the effective cross section in the denominator, which decreases as the photon energy fraction $\xi$ increases for $\xi<0.07$. Therefore, this behavior corroborates our result that the pocket formula cannot have a constant effective cross section.

As a matter of fact, any differences (other than a multiplicative factor) between Figs. 5 and 4 are due to the dependence of the effective cross section on $\xi$. In addition, the different lines in Fig. 5 presenting different behaviors as the fixed rapidity is changed is a direct result of fact that the photon $b$ distribution changes with $\xi$. In order to illustrate how the shape changes with rapidity, we have added an extra Fig. 6 showing the ratios of the differential cross section to a
Table 1 Table with the integrated cross sections for DPS and SPS production processes

\begin{tabular}{lcrr}
\hline$\sqrt{s}(\mathrm{TeV})$ & 8.16 & 50 & \multicolumn{1}{c}{100} \\
\hline SPS UPC $c \bar{c}$ production in mb & & \\
Direct & 3.10 & 10.46 & 15.75 \\
Resolved & 0.35 & 1.81 & 3.03 \\
Total & 3.45 & 12.27 & 18.78 \\
DPS UPC $c \bar{c} b \bar{b}$ production in nb & & \\
Total & 3.55 & 54.1 & 136 \\
\hline
\end{tabular}

given reference curve at fixed $y_{c}=0$ (left panel) and $y_{b}=0$ (right panel) corresponding left and right panels of Fig. 5, respectively. As expected, the largest deviations in shapes emerge at forward rapidities corresponding to large $\xi$. It is much easier to see this effect in our UPC example than in standard four-gluon DPS, since the photon impact-parameter distributions have a clearer and stronger dependence on the longitudinal momentum fraction as opposed to gluons. Just to clarify, we remark again that no correlations between the two photons were taken into account, in the sense that picking the first photon does not change the distribution of the second photon. I

In Fig. 7 we integrate over one more rapidity, leaving only $y_{b}$ or $y_{c}$ unintegrated. Together with Table 1, we see that we have a significant cross section, of the order of nanobarns, indicating that such an observable can be measured currently at the LHC and of course also at higher energy future colliders.

In the differential cross sections, e.g. shown in Figs. 4, 5 , and 7 , integrated over the antiquark rapidities, one can increase statistics by multiplying them by a factor of two (for SPS) or by the factor of four (for DPS) if a measure-
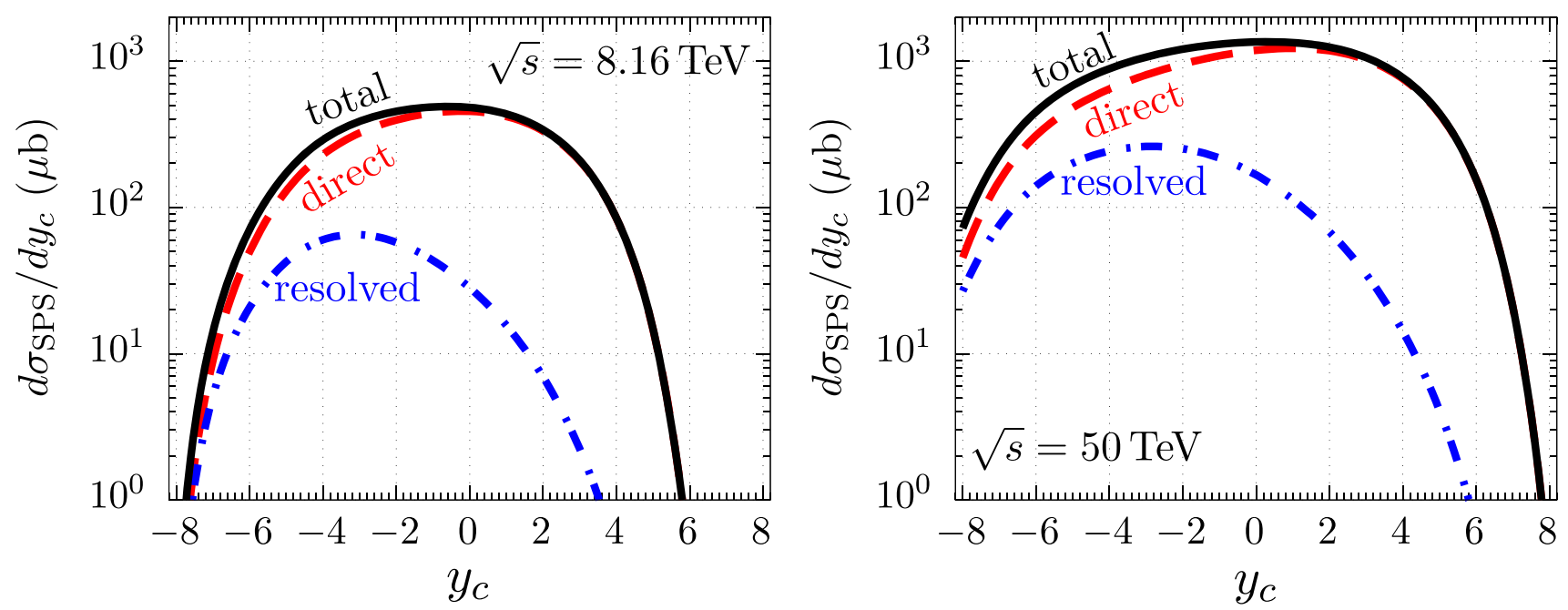

Fig. 4 The SPS $c \bar{c}$ quark production cross section in $p A$ UPCs as a function of rapidity $y_{c}$. Energy of $\sqrt{s}=8.16 \mathrm{TeV}$ is shown in the left panel and $\sqrt{s}=50 \mathrm{TeV}$ is shown in the right panel. The heavy ion is coming from the left 

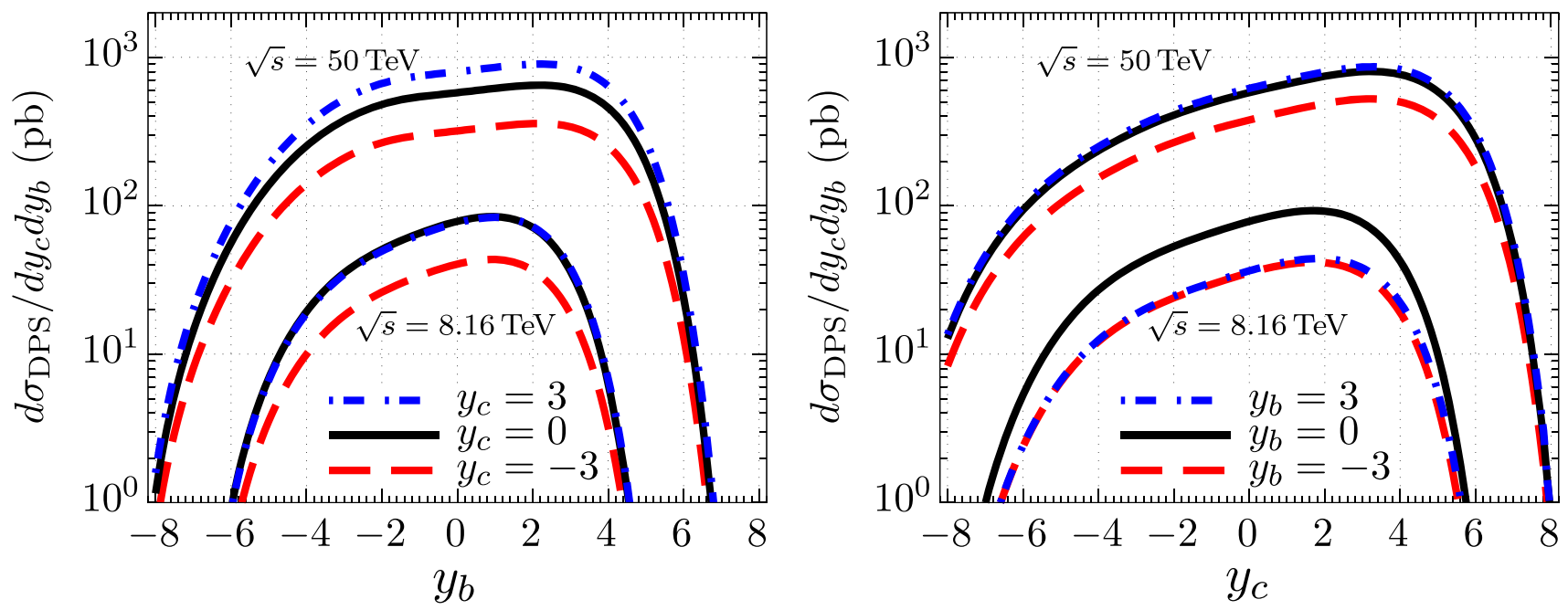

Fig. 5 The DPS $c \bar{c} b \bar{b}$ production cross section in $p A$ UPCs as a function of $c$-quark rapidity at fixed $b$ rapidity (left panel) and as a function of $b$-quark rapidity at fixed $c$-quark rapidity (right panel)
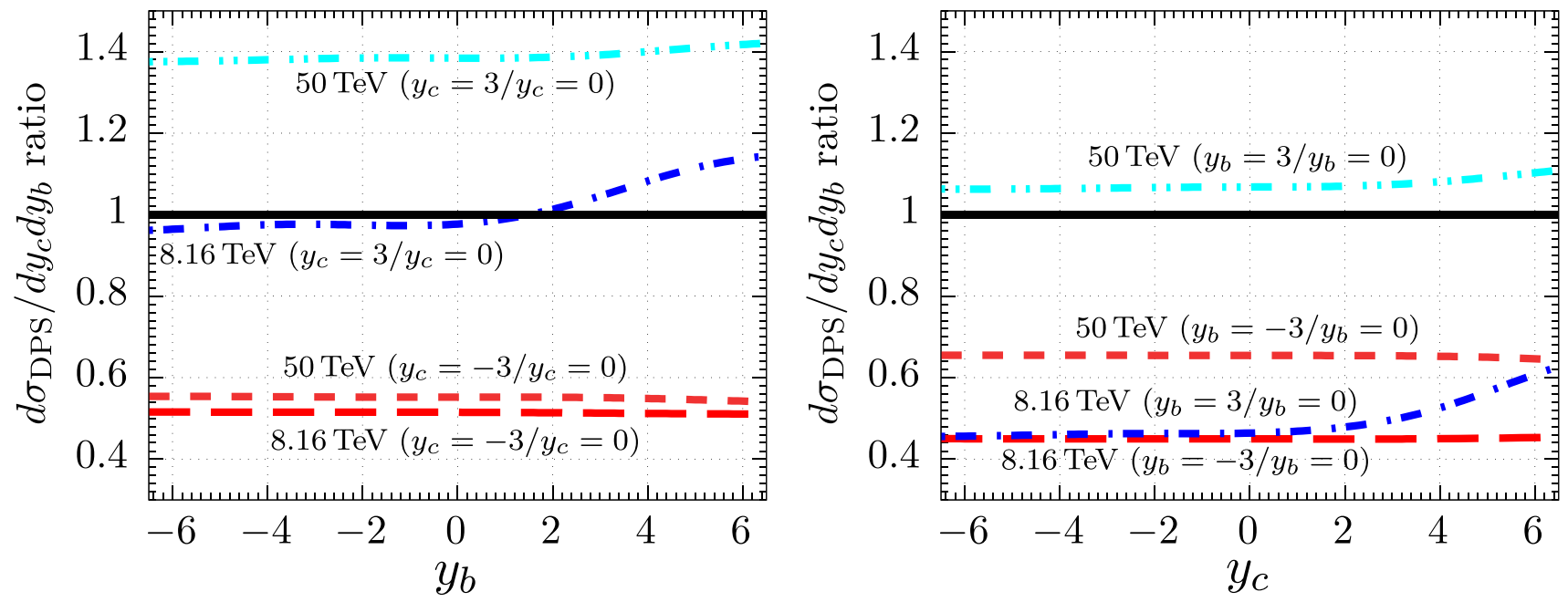

Fig. 6 The same as in Fig. 5 but for the ratios of the differential cross section at each fixed rapidity to a reference curve at fixed $y_{c}=0$ (left panel) and $y_{b}=0$ (right panel) and at each given energy

ment detects open heavy flavored mesons containing both heavy quarks and antiquarks. In this case, charged $D^{ \pm}$and $B^{ \pm}$mesons should be detected in the DPS final state, simultaneously ensuring that $D^{+} D^{-}$and $B^{+} B^{-}$meson pairs are produced at well separated rapidity domains to suppress the SPS $\gamma+g \rightarrow c \bar{c} b \bar{b}$ background contribution. In principle, for this purpose it suffices to consider the individual (anti)quark rapidities $y_{c}$ (or $y_{\bar{c}}$ ) and $y_{b}$ (or $y_{\bar{b}}$ ) to be far apart from each other.

\section{Conclusions}

In this paper we investigated the double parton interaction between heavy ion and proton in ultraperipheral collisions.
For the main contribution, where two photons from the heavy ion interact with two gluons from the proton, we developed a new pocket formula, with some peculiarities when compared with the usual one. In our case, as the distribution of photons is not as localized in impact parameter as the gluon distribution, the effective cross section is rather large, roughly dozens of barns. Another consequence is that the effective cross section is heavily dependent on the photon longitudinal momentum fraction, and this cannot be neglected. Therefore, we do not have a simple multiplication of SPS cross sections, but instead a convolution in the photon longitudinal momentum fraction.

We presented our results in terms of the cross section to produce $c$ and $b$ quarks as a function of rapidities. In this way, we can assert that each heavy quark gives us information 

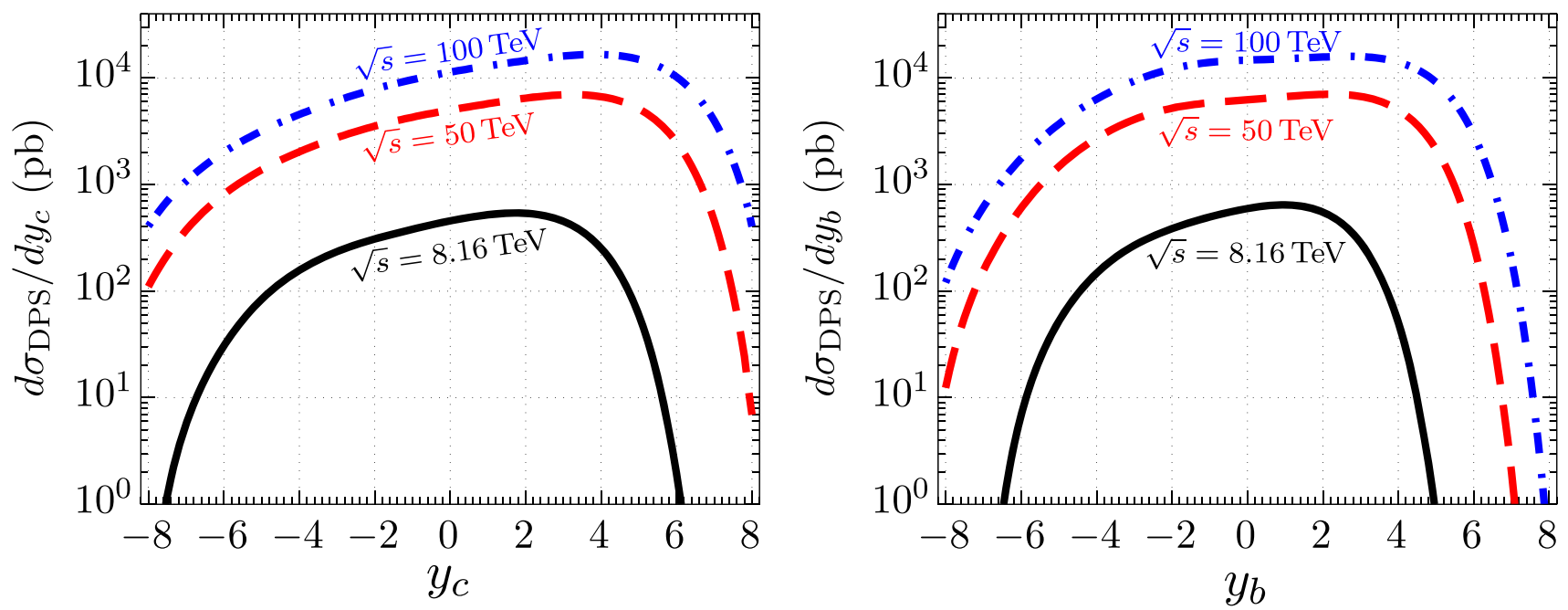

Fig. 7 DPS $c \bar{c} b \bar{b}$ production cross section in $p A$ UPCs as a function of $c$ rapidity with $y_{b}$ integrated (left panel) and as a function of $b$-quark rapidity with $y_{c}$ integrated (right panel)

about one of the gluons in the initial state. Therefore, this is an effective and direct probe of the double-gluon distribution that can be studied at the HL-LHC or at a future collider, e.g. at $50 \mathrm{TeV}$, for which the predictions are shown in Figs. 5 and 7.

We point out that the most efficient way of suppressing the SPS $\gamma+g \rightarrow c \bar{c} b \bar{b}$ background contribution is to measure the open charm and open bottom mesons at large relative rapidity separation of a few units. So, future measurements aiming at precision measurement of the DPS contribution in the considered process are encouraged to have the corresponding detectors covering different rapidity domains of the phase space (for example, the central and forward/backward cases).

Acknowledgements This work was supported by Fapesc, INCT-FNA (464898/2014-5), and CNPq (Brazil) for EGdO and EH. This study was financed in part by the Coordenação de Aperfeiçoamento de Pessoal de Nível Superior-Brasil (CAPES)—Finance Code 001. The work has been performed in the framework of COST Action CA15213 "Theory of hot matter and relativistic heavy-ion collisions" (THOR). R.P. is supported in part by the Swedish Research Council grants, contract numbers 621-2013-4287 and 2016-05996, by CONICYT grant MEC80170112, as well as by the European Research Council (ERC) under the European Union's Horizon 2020 research and innovation programme (grant agreement No 668679). This work was also supported in part by the Ministry of Education, Youth and Sports of the Czech Republic, project LT17018.

Data Availability Statement This manuscript has no associated data or the data will not be deposited [Authors' comment: There is no associated data to this manuscript.]

Open Access This article is distributed under the terms of the Creative Commons Attribution 4.0 International License (http://creativecomm ons.org/licenses/by/4.0/), which permits unrestricted use, distribution, and reproduction in any medium, provided you give appropriate credit to the original author(s) and the source, provide a link to the Creative
Commons license, and indicate if changes were made. Funded by SCOAP ${ }^{3}$.

\section{References}

1. N. Paver, D. Treleani, Il Nuovo Cimento A (1965-1970) 70, 215 (1982), ISSN 1826-9869. https://doi.org/10.1007/BF02814035

2. M. Mekhfi, Phys. Rev. D 32, 2371 (1985)

3. T. Sjöstrand, M. van Zijl, Phys. Rev. D 36, 2019 (1987). https:// doi.org/10.1103/PhysRevD.36.2019

4. J.R. Gaunt, W.J. Stirling, JHEP 03, 005 (2010). arXiv:0910.4347

5. M. Diehl, D. Ostermeier, and A. Schafer, JHEP 03, 089 (2012). [Erratum: JHEP03,001(2016)], arXiv:1111.0910

6. A.V. Manohar, W.J. Waalewijn, Phys. Rev. D 85, 114009 (2012). https://doi.org/10.1103/PhysRevD.85.114009

7. R. Aaij et al., (LHCb), JHEP 07, 052 (2016)

8. B. Blok, M. Strikman, Eur. Phys. J. C 76, 694 (2016). arXiv:1608.00014

9. M. Rinaldi, S. Scopetta, M. Traini, V. Vento, Phys. Lett. B 752, 40 (2016). arXiv: 1506.05742

10. R. Maciuła, A. Szczurek, Phys. Rev. D 97, 094010 (2018). https:// doi.org/10.1103/PhysRevD.97.094010

11. F. Abe, M. Albrow, D. Amidei, C. Anway-Wiese, G. Apollinari, M. Atac, P. Auchincloss, P. Azzi, A.R. Baden, N. Bacchetta et al., Phys. Rev. D 47, 4857 (1993). https://doi.org/10.1103/PhysRevD. 47.4857

12. M. Aaboud et al. (ATLAS), Phys. Lett. B 790, 595 (2019). [Phys. Lett.790,595(2019)]. arXiv:1811.11094

13. G. Calucci, D. Treleani, Phys. Rev. D 60, 054023 (1999). https:// doi.org/10.1103/PhysRevD.60.054023

14. M. Diehl, J.R. Gaunt, Adv. Ser. Direct. High Energy Phys. 29, 7 (2018). arXiv: 1710.04408

15. H.-M. Chang, A.V. Manohar, W.J. Waalewijn, Phys. Rev. D 87, 034009 (2013a). https://doi.org/10.1103/PhysRevD.87.034009

16. M. Rinaldi, S. Scopetta, V. Vento, Phys. Rev. D 87, 114021 (2013). https://doi.org/10.1103/PhysRevD.87.114021

17. M. Rinaldi, S. Scopetta, M. Traini, V. Vento, JHEP 12, 028 (2014). arXiv: 1409.1500 
18. T. Åkesson, M. Albrow, S. Almehed, O. Benary, H. Bøggild, O. Botner, H. Breuker, A. Carter, J. Carter, Y. Choi, et al., Zeitschrift für Physik C Part. Fields 34, 163 (1987), cited By 140. https://www.scopus.com/inward/record.uri?eid=2-s2. 0-25044475120\&doi $=10.1007 \% 2 \mathrm{fBF} 01566757 \&$ partnerID $=40 \&$ md5=eed609a5f61d17f88a971070778a3381

19. J. Alitti, G. Ambrosini, R. Ansari, D. Autiero, P. Bareyre, I. Bertram, G. Blaylock, P. Bonamy, K. Borer, M. Bourliaud, et al., Phys. Lett. B 268, 145 (1991), ISSN 0370-2693. http://www. sciencedirect.com/science/article/pii/037026939190937L

20. The LHCb collaboration, R. Aaij, C. Abellan Beteta, B. Adeva, M. Adinolfi, C. Adrover, A. Affolder, Z. Ajaltouni, J. Albrecht, F. Alessio, J. High Energy Phys. et al., 141 (2012). ISSN 1029-8479 (2012). https://doi.org/10.1007/JHEP06(2012)141

21. V.M. Abazov, B. Abbott, M. Abolins, B.S. Acharya, M. Adams, T. Adams, E. Aguilo, G.D. Alexeev, G. Alkhazov, A. Alton et al., The D0 Collaboration. Phys. Rev. D 81, 052012 (2010). https:// doi.org/10.1103/PhysRevD.81.052012

22. G. Aad et al., ATLAS. Eur. Phys. J. C 75, 229 (2015). arXiv: 1412.6428

23. R. Aaij et al. (LHCb), JHEP 06, 047 (2017), [Erratum: JHEP10,068(2017)]. arXiv:1612.07451

24. A.M. Sirunyan et al., (CMS), JHEP 02, 032 (2018). arXiv: 1712.02280

25. M. Diehl and A. Schäfer, Physics Letters B 698, 389 (2011), ISSN 0370-2693. http://www.sciencedirect.com/science/article/ pii/S0370269311002863

26. Multi-Parton Interactions at the LHC (2011). arXiv:1111.0469

27. S. Bansal et al., in Workshop on Multi-Parton Interactions at the LHC (MPI @ LHC 2013) Antwerp, Belgium, December 2-6, 2013 (2014). arXiv:1410.6664

28. M. Łuszczak, R. Maciuła, A. Szczurek, Phys. Rev. D 85, 094034 (2012). https://doi.org/10.1103/PhysRevD.85.094034

29. The LHCb collaboration, R. Aaij, C. A. Beteta, B. Adeva, M. Adinolfi, C. Adrover, A. Affolder, Z. Ajaltouni, J. Albrecht, F. Alessio, J. High Energy Phys. et al., 108 (2014). ISSN 1029-8479 (2014). https://doi.org/10.1007/JHEP03(2014)108

30. R. Maciuła, A. Szczurek, Phys. Rev. D 87, 074039 (2013). https:// doi.org/10.1103/PhysRevD.87.074039

31. A. Del Fabbro, D. Treleani, Phys. Rev. D 66, 074012 (2002). https:// doi.org/10.1103/PhysRevD.66.074012
32. E.R. Cazaroto, V.P. Gonçalves, F.S. Navarra, Phys. Rev. D 88, 034005 (2013). https://doi.org/10.1103/PhysRevD.88.034005

33. R. Maciuła, A. Szczurek, Phys. Rev. D 96, 074013 (2017). https:// doi.org/10.1103/PhysRevD.96.074013

34. V.M. Abazov et al., (D0), Phys. Rev. D 90, 111101 (2014). arXiv: 1406.2380

35. V. M. Abazov et al. (D0), Phys. Rev. Lett. 116, 082002 (2016). arXiv: 1511.02428

36. M. Aaboud et al. (ATLAS), Eur. Phys. J. C 77, 76 (2017). arXiv: 1612.02950

37. V. Khachatryan et al. (CMS), JHEP 05, 013 (2017). arXiv: 1610.07095

38. H. Mäntysaari, B. Schenke, Phys. Rev. Lett. 117, 052301 (2016). arXiv:1603.04349

39. S.R. Klein, J. Nystrand, R. Vogt, Phys. Rev. C 66, 044906 (2002). arXiv:hep-ph/0206220

40. A. Adeluyi, T. Nguyen (2012). arXiv:1210.3327

41. C.F. von Weizsacker, Z. Phys. 88, 612 (1934)

42. E.J. Williams, Phys. Rev. 45, 729 (1934)

43. M. Kłusek-Gawenda, A. Szczurek, Phys. Rev. C 82, 014904 (2010). https://doi.org/10.1103/PhysRevC.82.014904

44. L. Frankfurt, M. Strikman, C. Weiss, Phys. Rev. D 83, 054012 (2011). arXiv:1009.2559

45. S. Dulat, T.-J. Hou, J. Gao, M. Guzzi, J. Huston, P. Nadolsky, J. Pumplin, C. Schmidt, D. Stump, C.P. Yuan, Phys. Rev. D 93, 033006 (2016). arXiv: 1506.07443

46. P. Aurenche, M. Fontannaz, J.P. Guillet, Eur. Phys. J. C 44, 395 (2005). arXiv:hep-ph/0503259

47. B. Blok, Yu. Dokshitser, L. Frankfurt, M. Strikman, Eur. Phys. J. C 72, 1963 (2012). arXiv:1106.5533

48. B. Blok, Yu. Dokshitzer, L. Frankfurt, M. Strikman, Eur. Phys. J. C 74, 2926 (2014). arXiv:1306.3763

49. H.-M. Chang, A.V. Manohar, W.J. Waalewijn, Phys. Rev. D 87, 034009 (2013b). arXiv:1211.3132 